\title{
Research on control strategy for AC side asymmetric fault of MMC-HVDC transmission system
}

\author{
Mingguang Zhang, Huzhong Sun*, Richang Guo, and Yao Shen \\ College of Electrical and Information Engineering, Lanzhou University of Technology, China
}

\begin{abstract}
When the Modular Multilevel Converter (MMC) fails on the $\mathrm{AC}$ side, the operating characteristics of MMC will be damaged. If the system wants to retain stable for a long time in the process of operation, it is obliged to design the control strategy in case of asymmetric fault. In this article, the control strategy based on feedback linearization principle is adopted to diminish the negative sequence current caused by breakdown, and a DC voltage controller is devised to suppress the fluctuation of the double frequency component under asymmetric fault conditions. The validity of the control strategy requires to be verified, the running results on PSCAD platform indicate this method is effective and feasible.
\end{abstract}

\section{Introduction}

Due to the increasing demand for electricity, the traditional power supply mode is difficult to meet people's greater demand for power resources. The power transmission system which has advantage of capacity, highly safety and efficiency. These merits determine that it will be used in the power grid. The development of flexible DC transmission technology [1-2] solves the power supply problems under special conditions, such as island power supply and offshore wind farm access to the grid, which makes largescale and long-distance transmission possible. The modular multilevel converter is composed of several sub modules in series [3-4]. The voltage can be adjusted by turning on and off the electronic device. Because of its superior performance, it has been widely used in many conditions. A control method is employed to make the mmc-hvdc system operate normally in [5-6], so that the system can reach stability faster. The circulating current in the arm of MMC during operation is analyzed and a control method is designed to suppress the circulating current component in [7-9]. A method is employed to reduce the fluctuation of current and power in [10]. The sequence components on the AC side are segregated into diverse sections, and the change process of AC and DC side current is analyzed in [11]. At the same time, the corresponding negative sequence current control method is proposed to reduce the fluctuation range of module voltage and improve the stability of system operation. The second order generalized integrator can speedily separate the sequence components into separate sections. [12]. A reduced order resonant regulator is adopted to

\footnotetext{
*Corresponding author: 2775214955@qq.com
} 
quickly separate the positive and negative sequence components in [13-14]. Aiming at the issues of AC side fault in MMC system, the feedback linearization method is adopted to project the inner loop current circuit, which can restrain the negative sequence current to a certain extent. At the same time, a DC voltage zero sequence compensator is designed to reduce the fluctuation of DC side voltage and current and improves the operation characteristics of the system when asymmetric fault occurs.

\section{Analysis of MMC equivalent circuit}

The schematic wiring diagram of modular multi electric equal value is drawn here. The bridge arm is linked with lots of modules. $i_{p j}$ and $i_{n j}$ are the currents flowing through the upper and lower bridge arms respectively. $U_{p j}$ and $U_{n j}$ represent the voltage of each cell separately. $u_{s j}$ is AC side voltage and $i_{s j}$ is AC side current. $U_{d c}$ is DC side voltage and $I_{d c}$ is the corresponding current. $R_{0}$ and $L_{0}$ are bridge arm resistance and reactance separately. The bridge arm reactor is able to suppress the current as a result of instantaneous voltage imbalance, but adding the filter reactor will reduce the circulating current effect and increase the complexity of the system. MMC module has a high degree of integration. In the process of operation, the on-off signal is applied to the controller to control the on-off of the electronic devices linked in the system. The number of sub modules has a certain effect of the waveform of output voltage. In actual use, the number of sub modules can be appropriately increased to improve the accuracy.

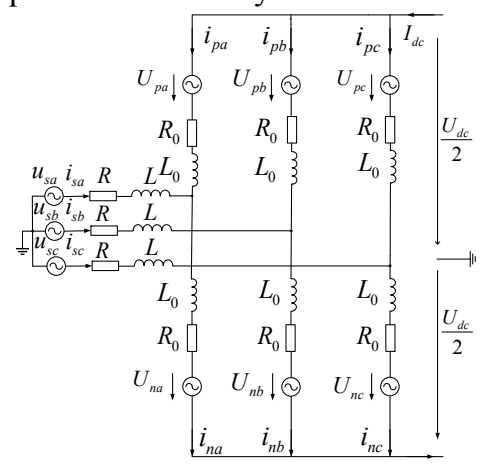

Fig. 1. Modular multilevel simplified equivalent circuit

\section{Asymmetric fault control strategy}

\subsection{Mathematical model of MMC under asymmetric condition}

When the MMC AC side system has asymmetric fault, it will engender negative sequence current. This ingredient will give arise to the asymmetry of system electrical parameters. Therefore, it is obliged to take relevant control strategies. The appropriate model contributes to analyse the system. The asymmetric components can be represented by the equivalent models of positive sequence and negative sequence. The figure below shows the equivalent diagram under two order components. 

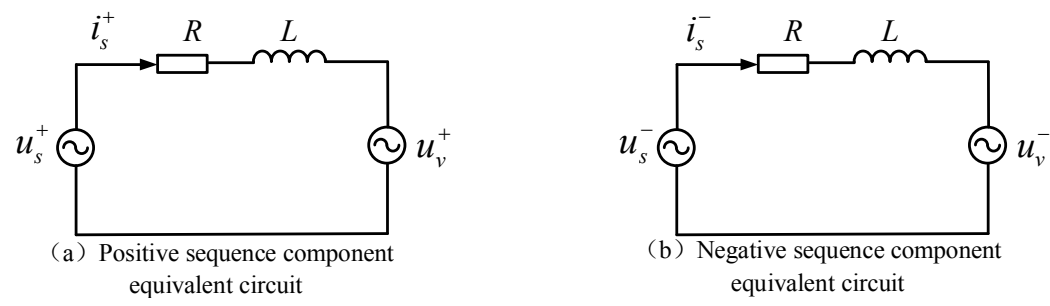

Fig. 2. Equivalent circuit diagram of sequence components

Through the analysis of the simplified modular multilevel equivalent circuit, the mathematical expressions of grid side and converter side of the system are easily obtained.

$$
u_{s j}-u_{v j}=L \frac{d i_{s j}}{d t}+R i_{s j}
$$

Through a series of coordinate transformation of equation (1), we can get the expression of positive and negative sequence voltage mathematical model in $\mathrm{d}$ and q coordinate system as follows.

$$
\begin{aligned}
& {\left[\begin{array}{c}
L \frac{d i_{s d}^{+}}{d t} \\
L \frac{d i_{s q}^{+}}{d t}
\end{array}\right]=\left[\begin{array}{cc}
-R & \omega L \\
-\omega L & -R
\end{array}\right]\left[\begin{array}{l}
i_{s d}^{+} \\
i_{s q}^{+}
\end{array}\right]+\left[\begin{array}{l}
u_{s d}^{+} \\
u_{s q}^{+}
\end{array}\right]-\left[\begin{array}{l}
u_{v d}^{+} \\
u_{v q}^{+}
\end{array}\right]} \\
& {\left[\begin{array}{c}
L \frac{d i_{s d}^{-}}{d t} \\
L \frac{d i_{s q}^{-}}{d t}
\end{array}\right]=\left[\begin{array}{cc}
-R & -\omega L \\
\omega L & -R
\end{array}\right]\left[\begin{array}{l}
i_{s d}^{-} \\
i_{s q}^{-}
\end{array}\right]+\left[\begin{array}{l}
u_{s d}^{-} \\
u_{s q}^{-}
\end{array}\right]-\left[\begin{array}{l}
u_{v d}^{-} \\
u_{v q}^{-}
\end{array}\right]}
\end{aligned}
$$

\subsection{Mathematical model analysis and positive and negative sequence current controller design}

The transformed positive and negative sequence components are independent of each other. The sequence component current controller can be received by analysis, and the mathematical formula of low frequency transient can be obtained by rewriting equations (2) and (3).

$$
\begin{aligned}
& \left\{\begin{array}{l}
\frac{d i_{s d}^{+}}{d t}=\frac{-R i_{s d}^{+}}{L}+\omega i_{s q}^{+}+\frac{u_{s d}^{+}}{L}-\frac{u_{v d}^{+}}{L} \\
\frac{d i_{s q}^{+}}{d t}=\frac{-R i_{s q}^{+}}{L}-\omega i_{s d}^{+}+\frac{u_{s q}^{+}}{L}-\frac{u_{v q}^{+}}{L}
\end{array}\right. \\
& \left\{\begin{array}{l}
\frac{d i_{s d}^{-}}{d t}=\frac{-R i_{s d}^{-}}{L}-\omega i_{s q}^{-}+\frac{u_{s d}^{-}}{L}-\frac{u_{v d}^{-}}{L} \\
\frac{d i_{s q}^{-}}{d t}=\frac{-R i_{s q}^{-}}{L}+\omega i_{s d}^{-}+\frac{u_{s q}^{-}}{L}-\frac{u_{v q}^{-}}{L}
\end{array}\right.
\end{aligned}
$$

In the decoupling process of the above formula, some suitable additional equations are joined to simplify the whole process. The equation is below. 


$$
\begin{aligned}
& \left\{\begin{array}{l}
\frac{d i_{s d}^{+}}{d t}+\lambda i_{s d}^{+}=\lambda i_{s d r e f}^{+} \\
\frac{d i_{s q}^{+}}{d t}+\lambda i_{s q}^{+}=\lambda i_{s q r e f}^{+}
\end{array}\right. \\
& \left\{\begin{array}{l}
\frac{d i_{s d}^{-}}{d t}+\mu i_{s d}^{-}=\mu i_{s d r e f}^{-} \\
\frac{d i_{s q}^{-}}{d t}+\mu i_{s q}^{-}=\mu i_{s q r e f}^{-}
\end{array}\right.
\end{aligned}
$$

In equations (6) and (7), $\lambda$ and $\mu$ are the parameters of PI controller. By introducing equations (7) and (6) into equations (5) and (4), the mathematical model of each sequence current controller after decoupling may be gained.

$$
\begin{gathered}
\left\{\begin{array}{l}
u_{v d}^{+}=u_{s d}^{+}-R i_{s d}^{+}+\omega L i_{s q}^{+}-\lambda L\left(i_{s d r e f}^{+}-i_{s d}^{+}\right) \\
u_{v q}^{+}=u_{s q}^{+}-R i_{s q}^{+}-\omega L i_{s d}^{+}-\lambda L\left(i_{s q r e f}^{+}-i_{s q}^{+}\right)
\end{array}\right. \\
\left\{\begin{array}{l}
u_{v d}^{-}=u_{s d}^{-}-R i_{s d}^{-}-\omega L i_{s q}^{-}-\mu L\left(i_{s d r e f}^{-}-i_{s d}^{-}\right) \\
u_{v q}^{-}=u_{s q}^{-}-R i_{s q}^{-}+\omega L i_{s d}^{-}-\mu L\left(i_{s q r e f}^{-}-i_{s q}^{-}\right)
\end{array}\right.
\end{gathered}
$$

According to the analysis of the decoupling mathematical model, the block diagram of the inner loop positive and negative sequence current controller is designed which appears in the figure below.
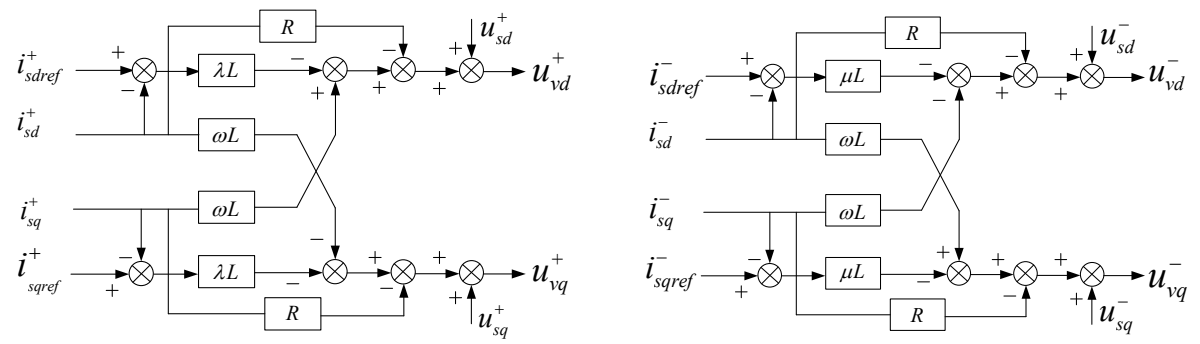

Fig. 3. Decoupling control chart of positive and negative sequence

$i_{\text {sdref }}^{+}$is the reference value of d-axis positive sequence current component and $i_{\text {sqref }}^{+}$is the corresponding q-axis component. $i_{\text {sdref }}^{-}$and $i_{\text {sqref }}^{-}$represent the negative sequence reference values of $\mathrm{d}$-axis and q-axis respectively. When single-phase short circuit occurs, $i_{\text {sdref }}^{-}$and $i_{\text {sqref }}^{-}$are set to $0 . i_{\text {sdref }}^{+}$and $i_{\text {sqref }}^{+}$are determined according to the active control circuit and reactive control circuit. Under the above control, the negative sequence current is eliminated with modulation algorithm.

\subsection{DC voltage control strategy}

The instantaneous value of bridge arm power is composed of DC component, double frequency negative sequence and zero sequence component. The DC component provides voltage for DC side bus and plays the role of power transmission. The negative sequence component is controlled by the negative sequence current controller. The zero sequence component of bridge arm power will create a range of fluctuations. In order to suppress this 
kind of fluctuation, it is necessary to compensate the zero sequence component and design the corresponding control strategy to reduce the second harmonic fluctuation.

$$
\left[\begin{array}{c}
U_{a}^{0} \\
U_{b}^{0} \\
U_{c}^{0}
\end{array}\right]=\frac{1}{3}\left[\begin{array}{ccc}
2 & -1 & -1 \\
-1 & 2 & -1 \\
-1 & -1 & 2
\end{array}\right]\left[\begin{array}{c}
U_{a} \\
U_{b} \\
U_{c}
\end{array}\right]
$$

The compensation component of the zero sequence voltage can be obtained by passing the generated zero sequence voltage through the zero sequence compensator. The compensation component is applied to the voltage reference command, and the corresponding trigger pulse is generated by the modulation algorithm. From the above analysis, the structure diagram of DC voltage fluctuation controller can be drawn.

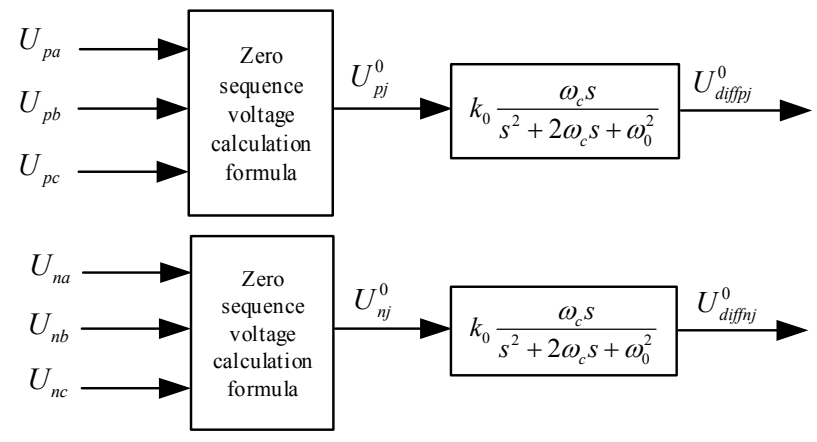

Fig. 4. DC voltage fluctuation control chart

By combining the two parts and combining with the appropriate modulation algorithm, the overall framework control chart of MMC can be obtained.

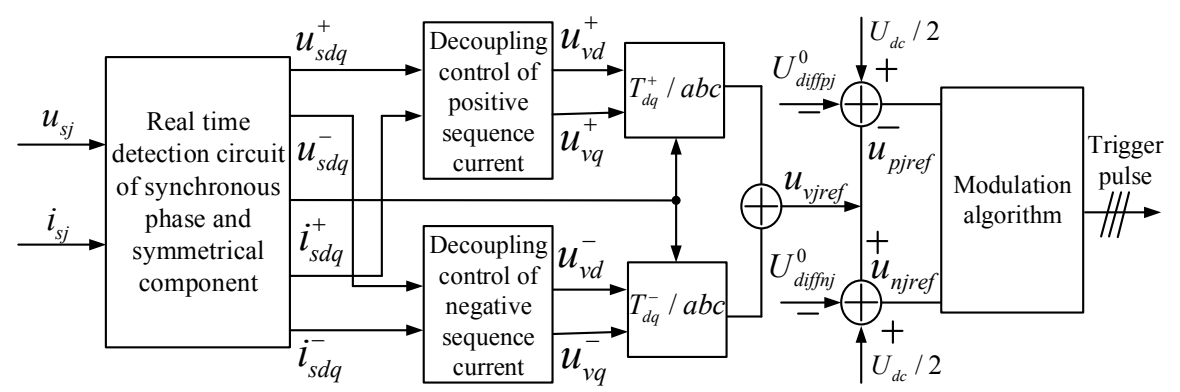

Fig. 5. Overall control chart of MMC under unbalanced voltage

\section{Simulation validation}

The validity of the designed control strategy requires to be tested when asymmetric fault occurs in the system. It is obliged to build MMC-HVDC on PSCAD / EMTDC platform. The rectifier side is dominated by constant DC voltage and reactive power, while the inverter side is regulated by constant active power and reactive power. The major system parameters are listed down below. 
Table 1. MMC-HVDC Transmission system parameter table

\begin{tabular}{|c|c|}
\hline Parameter & Numerical value \\
\hline AC side voltage $/ \mathrm{kV}$ & 220 \\
\hline DC side bus voltage/kV & 330 \\
\hline $\begin{array}{c}\text { Number of single bridge } \\
\text { arm submodules } / \mathrm{N}\end{array}$ & 20 \\
\hline Submodule capacitance/mF & 4.7 \\
\hline Transformer capacity/MVA & 570 \\
\hline $\begin{array}{c}\text { Bridge arm equivalent } \\
\text { reactance/H }\end{array}$ & 0.1 \\
\hline
\end{tabular}

It is assumed that the single-phase ground fault arises at the AC side of the system in $3 \mathrm{~S}$.The fault is cleared after $0.15 \mathrm{~s}$, and the fault phase is selected as phase A. Figure 6 shows that the phase A voltage basically drops to 0 , and the voltage returns to normal after clearing the fault. Figure 7 shows the output current waveform of the AC side of the power grid. Under the action of the current controller, the negative sequence current is controlled, and the three-phase current remains symmetrical. Figure 8 and Figure 9 are the simulation diagrams of DC side voltage and current after the controller is put into operation. The voltage and current fluctuations decrease after the controller is put into operation, which shows that the designed controller can improve the operation characteristics of the system and improve the stability of the system in the case of asymmetric fault.

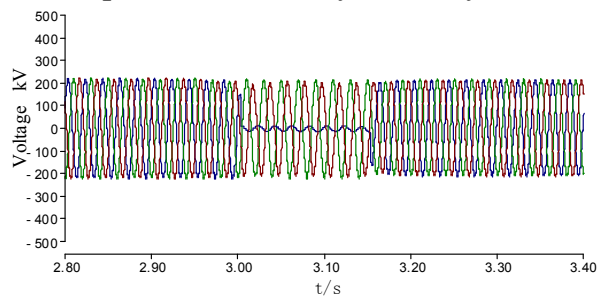

Fig. 6. Three phase voltage waveform of AC side

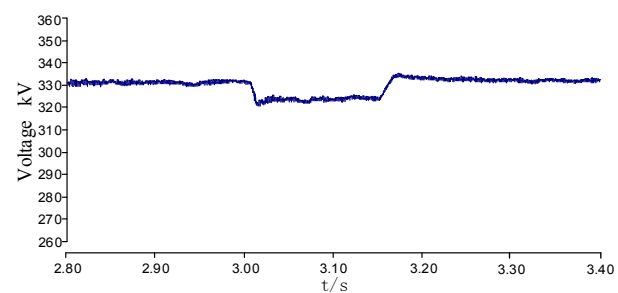

Fig. 8. DC side voltage when put into controller

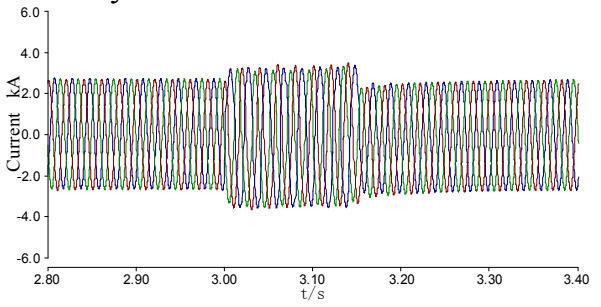

Fig. 7. Output current waveform of AC side

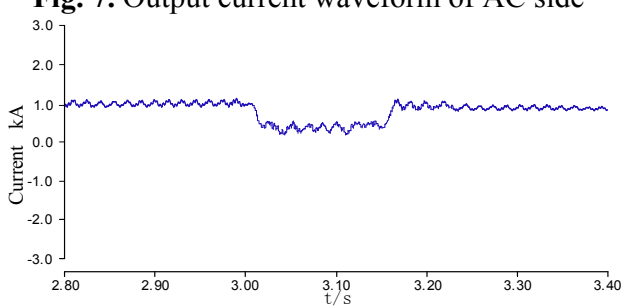

Fig. 9. DC side current when put into controller

\section{Conclusion}

Asymmetric fault will destroy the stability of MMC system. This paper designs a strategy under asymmetric fault to improve this situation. Through the design of the corresponding current controller, the negative sequence current component is reduced, and then the voltage compensation method is adopted to decrease the frequency doubling fluctuation of the DC side electrical component. This strategy has a certain engineering application value.

This work is supported by the National Natural Science Foundation of China (51567016). 


\section{References}

1. XU Zheng, XUE Yinglin, Zhang Zheren. VSC-HVDC Technology Suitable for Bulk Power Overhead Line Transmission[J]. Proceedings of the CSEE, 2014,34(29):50515062.

2. YUE Bo MEI Nian LIU Si-yuan CHEN Dong ZHANG Shu-jun .Overview of HVDC Flexible [J].China Electric Industry (Technical Edition) ,2014(05):43-47.

3. Chu Y, Huang S, Kong F, et al. Steady State Control of MMC-HVDC System Based on Internal Model Controller[J]. Power System Technology, 2015, 39(8): 2223-2229.

4. Wang Yang,Aksoz Ahmet,Geury Thomas,Ozturk Salih Baris,Kivanc Omer Cihan,Hegazy Omar. A Review of Modular Multilevel Converters for Stationary Applications[J]. Applied Sciences,2020,10(21).

5. Sun Yiying, Zhao Chengyong, Zhao Jing, Zhang Jianpo.A Steady-State Control Strategy of MMC-HVDC Transmission System Based on Two-Phase Stationary Reference Frame [J]. Power System Technology, 2013,37(05) :1384-1388.

6. FU Hua, CAO Qingchun. SSPFM Control Strategy for Capacitance Equalizing the MMC-HVDC System Based on Model Predictions[J].High Voltage Engineering,2017,43(04):1114-1120.

7. ZHANG Lei, FAN Caiyun, HAN Kun, ZHANG Zhigang, SI Zhilei. MMC-HVDC precise circulation suppression control under asymmetrical network voltage[J]. Power System Protection and Control,2018,46(12) :42-49.

8. XUE Hua, LI Yang, WANG Yufei, YANG Xingwu, LIU Weidong. Passivity-based PI stability controland circulating current suppression method of MMC-HVDC[J]. Power System Protection and Control,2017,45(19) :78-85.

9. ZHANG Lei, FAN Caiyun, HAN Kun, ZHANG Zhigang, SI Zhilei. MMC-HVDC precise circulation suppression control under asymmetrical network voltage[J]. Power System Protection and Control, 2018,46(12): 42-49.

10. WANG Zhenhao, ZHANG Zhen ,LI Guoqing. An Unbalanced Fault Control Strategy for MMC-HVDC System Based on Compensation Principle[J]. Automation of Electric Power Systems, 2017,41(17): 94-100.

11. MA Shiqiang, YU Lixia, ZHENG Lianqing. Characteristics Analysis of MMC-HVDC AC Asymmetric Fault and Protection Strategy[J]. Journal of North China Electric Power University (Natural Science Edition) ,2015,42(06): 35-42+63.

12. Xue Shangqing, Cai Jinding.Detection of fundamental positive and negative sequence components based on second-order generalized integrator[J]. Power automation equipment,2011,31(11):69-73.

13. GUO Xiao-qiang, WU Wei-yang, CHEN Zhe. Multiple-complex coefficient-filterbased phase-locked loop and synchronization technique for three-phase grid-interfaced converters in distributed utility networks[J]. IEEE Transon Industrial Electronics, 2011, 58(4): 1194-1204.

14. Xie Hua, Zhao Xin, Shen Shen. Positiveand negative-sequence component detecting method based on reduced order resonant regulator[J]. Power System Protection and Control, 2013,41(14): 41-47. 